\title{
Clinical trials in chronic liver disease - What do they achieve?
}

\author{
Jenny Heathcote MD
}

$\mathrm{T}^{\mathrm{s}}$ he past 30 years have seen rapid growth in the number of clinical trials in liver disease; due mostly to effective drug discovery programs by the pharmaceutical industry. The advantages associated with therapeutic trials in chronic liver disease, or any other disease for that matter, go far beyond potential benefit to the individual patient, other beneficiaries include the treating physician, the sponsoring agency and their investors, the institution/university and the general public. But there is always a downside to any experiment. The disadvantages and advantages of clinical trials in liver disease are the topics for this discussion.

\section{THE INDIVIDUAL PATIENT}

Most clinical trials involve the assessment of a new therapeutic option, most often a drug but occasionally a device or surgical manoeuver. Individuals who are willing to participate in clinical studies generally do so because participation provides an opportunity to increase their therapeutic options. During the recruitment process, patients increase their understanding of their disease and often feel they benefit from closer monitoring (generally a necessary part of participating in any trial). Many patients also enjoy the fact that they feel they are making a contribution to medical science.

However, most clinical trials require that patients undergo a rigorous screening process, which may mean multiple hospital visits and lead to being told that they are an 'unsuitable' candidate for the study. There is no way of escaping a sense of rejection when this occurs. It is essential that an alternative option be readily available and that appropriate follow-up be maintained. For those who fulfill enrolment criteria, there is always the anxiety that goes with the unknown therapeutic potential of what is undergoing trial or of being randomized to placebo. Possible adverse events are another concern - in the field of liver disease, the fialuridine disaster is forever imprinted in our memories. To prevent such untoward occurrences, close monitoring is required but this can become very tedious and inconvenient over the ensuing months, and in some instances, even years. Patients soon begin to realize why it is important to have good veins, as repeated blood samples are a prerequisite for any clinical trial!

\section{THE TREATING PHYSICIAN}

\section{Pharmaceutical industry-sponsored studies}

There are many advantages to participating in multicentre pharmaceutical industry-sponsored studies, the most important being early access to potentially better therapies for patients with the added advantage of a treating nurse being part of the sponsorship. These are the most overt advantages to participating, but there are many more benefits that are not always taken advantage of. The financial reimbursement for the physicians' ongoing participation in performing clinical assessments of recruited patients can be put to excellent use in funding the infrastructure necessary to run a clinical research program - the more patients recruited, the bigger the 'slush fund'. Very often, if one becomes a major contributor it is likely that one or more publications in high impact journals will follow. It is necessary to participate in meetings to discuss the results and these may lead to the further collaboration with international colleagues. Participation in investigator meetings necessarily allows for exchange of ideas with colleagues and scientists within the industry, and sometimes leads to negotiations for subsequent investigator-initiated studies. Access to the huge databases that arise from these multicentre studies to allow one to examine a specific idea becomes negotiable once the major papers are published.

However, there are several disadvantages to participating in pharmaceutical industry-funded studies. Often the most troublesome issues are with contracts and the refusal of the industry to allow for concurrent studies. For those of us who are natural-born investigators, this always seems to be a huge waste of clinical material. As previously mentioned, it is advantageous to recruit large numbers of patients to reap maximum benefit. But this involves an enormous amount of work and a huge time commitment, both on the part of the treating nurses and the physicians. The demands of the licensing authorities seem to increase yearly, and this means volumes of paperwork.

Presented at Canadian Digestive Disease Week 2004 as the Canadian Association for the Study of the Liver gold medalist

University Health Network, Toronto Western Hospital, Toronto, Ontario

Correspondence: Dr Jenny Heathcote, University Health Network, Toronto Western Hospital, 399 Bathurst Street, Toronto, Ontario M5T 258.

Telephone 416-603-5914, fax 416-603-6281, e-mail jenny.heathcote@utoronto.ca

Received for publication June 8, 2004. Accepted June 8, 2004 
Just occasionally a study may be abruptly abandoned, sometimes for good reason (ie, presence of serious side effects and sometimes for such irritating things as bankruptcy!).

Inclusion criteria tend to be extremely rigid, which is why most industry studies cannot be readily generalized to the average patient in clinic. These rigid inclusion criteria necessarily mean a high rate of exclusion. The sense of rejection is not limited to the patient, and leaves an enormous sense of frustration for both the nurse and physician who have often spent considerable time organizing screening tests, the cost of which is rarely recovered.

\section{Investigator-initiated studies}

These give the investigator a chance to answer specific questions that particularly interest them. For this reason, they are much more preferable in terms of academic reward than most industry-sponsored studies. Only when one comes to designing a study does one realize that rigid inclusion criteria remain necessary. Thus, it is a prerequisite to have a large population base to allow recruitment of the required sample size. It may first be necessary to conduct a clinical study that allows one to locate a particular patient population. This is particularly relevant when the disease in question is rare (eg, primary biliary cirrhosis $[\mathrm{PBC}]$ ), or when the disease in question is one that is the interest of a wide range of specialists (eg, primary sclerosing cholangitis [PSC], a disease that may be monitored by luminal gastroenterologists and therapeutic endoscopists, as well as hepatologists). These so-called feasibility studies are required for many purposes. First, no funding agency will fund the study until they can be confident that the applicant does indeed have access to sufficient numbers of patients. Physicians in the community are not prepared to refer their patients to a referral centre unless there is a distinct advantage for their patient. Over the past 10 years or more, particularly since the start of the HIV epidemic, the involvement of affected patients has become essential for the approval and success of clinical research studies. It may take several years to establish a sense of trust both amongst the patient population and one's colleagues.

\section{THE 'DOMINO EFFECT'}

With a hindsight of 30 years, it is now possible to see quite clearly how one question may lead to another and then to another. With this experience it becomes easier to anticipate where a particular study may lead in the future and recruit the appropriate assistants sooner rather than later. For instance, Canada was just one of many countries that participated in randomized controlled studies of ursodeoxycholic acid (UDCA) in PBC. Had we recognized the potential back in 1988, we may have designed a common database. We did not, and thus we wasted a lot of time re-collecting the data when we decided to perform a combined analysis of our results. This effort was not wasted; we were then able to ask many more questions with the much larger sample sizes generated and, thus, we have continued collaborative ventures for the past 10 years. We have recruited additional sites, and we hope that with a new genetics consortium for PBC, we will be able to answer really important questions on the etiopathogenesis of this disease - a common database has already been developed.

\section{BEING SIDETRACKED}

Very often, it is the patients themselves who suggest the studies, and so it became obvious that fatigue was a major concern of many of our patients with liver disease, whether it be PBC, PSC or chronic hepatitis C. Because the symptom of fatigue is more in the realm of neurologists, neuropsychologists and psychiatrists, collaborative studies with a new group of colleagues was essential if we were to understand more about the pathogenesis of this symptom in our patient with liver disease. Again, the value of preliminary feasibility studies needs to be stressed - these preliminary studies can often be conducted by a summer student or a medical resident during his or her research elective. These 'cheap and cheerful' feasibility studies are exciting for the student and very helpful to the investigator.

\section{THE FRUSTRATIONS OF CLINICAL STUDIES}

Although one may consider a study design to be superb, things may go wrong that could never have been anticipated. The following examples illustrate two things: first, the importance of patient involvement in study design; and second, how too much information can be a bad thing! In the early 1990s, hormone replacement therapy (HRT) was being advocated as a cure for menopause and all its complications. However, estrogens promote cholestasis and, thus, it seemed appropriate to design a randomized controlled trial of HRT in postmenopausal women with $\mathrm{PBC}$ at risk for osteoporosis. This study was funded, and after 10 years of attempting to recruit the necessary sample size of 68 , we gave up! Over this period of time, 355 patients with $\mathrm{PBC}$ had been screened; only 91 were deemed eligible and only 31 agreed to be randomized. After randomization, nine of the 31 withdrew, leaving eight patients in the treatment group. Thus, a study that lasted nearly 10 years was eventually completed with 14 patients in the placebo group and eight patients in the treatment group! What we learned was that women who were in favour of HRT were already taking this medication and those who were not on HRT were mostly very antagonistic towards its use. The high withdrawal rate following randomization devastated our study; a similar withdrawal rate $(42 \%)$ also occurred in the Women's Health Initiative study but had less effect on the outcome because several thousands of individuals had been recruited. Somewhat later, we designed another study for individuals with congenital coagulation disorders infected with hepatitis $\mathrm{C}$ with or without coinfection with HIV. This study was designed to ensure that these individuals received appropriate therapy for their hepatitis $\mathrm{C}$ because they had been excluded by all the earlier pharmaceutical studies. However, recruitment was much harder than anticipated, mostly because the patient population, instead of being interested as their physicians had thought they were, had many misgivings about the study. Had we involved the affected community from the start I suspect that we may have had a much more successful outcome. 
It has become the law that all patients involved in clinical trials must be involved in the consent process. Whereas consent forms used to be quick and easy to read, they now involve several pages listing even the most esoteric of potential complications. These long lists are very daunting, particularly to those whose first language is not necessarily the language of the consent form. In a recent double-blind, randomized, cross over trial of ondansetron for fatigue in PBC, we explained to the patients that the drug had two major side effects, namely, constipation and headache. We were required to make a very careful record of these side effects. When the analyses were finally prepared, the results were very confusing. Whereas in the first period it appeared that fatigue improved to a similar degree in all patients, regardless of whether they were randomized to placebo or ondansetron, this was not the case in period two, where those who were then on placebo became more fatigued, and those randomized to ondansetron in this second period continued to improve. Examination of the data indicated that 'unblinding' due to the side effect of constipation had occurred during period two!

\section{WHAT TO DO WHEN YOU ARE 'STUMPED'}

Sometimes the results of studies leave one with nowhere to go, such as the ondansetron study for fatigue in $\mathrm{PBC}$ as described above. Under these circumstances, it may be valuable to go back to the drawing board, ie, take another look at pathogenesis. In the intervening years, several improvements in investigating neurocognitive behaviour and function have taken place. Perhaps it is appropriate to conduct studies using magnetic resonance spectroscopy and/or functional magnetic resonance imaging to examine the pathogenesis of fatigue in greater detail.

To date, we can only delay progression of PBC with UDCA therapy. We need to be able to prevent disease progression and induce regression. However, some patients with asymptomatic PBC may never progress, and epidemiological studies show that fewer than $50 \%$ of asymptomatic patients will die of their disease. Thus, we need to identify who is going to progress; to do this, we need to look more closely at the pathogenesis of this disease. Hematologists have already demonstrated that by examining molecular array patterns in patients with lymphoma, they can predict the subsequent course of disease. It is potentially possible that there is a molecular signature for disease progression in individuals with PBC. Clinical observation indicates that a large component of PBC is under genetic control. It is likely that many different genes are involved in the pathogenesis of this disease, but it may not be too hard to identify genes that govern response to therapy and disease progression.

\section{COLLABORATIVE VENTURES THAT LEAD TO OUTCOMES ONE WOULD NEVER HAVE DREAMT OF!}

Every cloud has a silver lining! So, although the therapeutic trial designed to help individuals with congenital coagulation disorders infected with hepatitis $\mathrm{C}$ with or without HIV in itself was not a success, the partnerships required to develop the treatment protocol led to new collaborations with colleagues in infectious diseases. Thus, when the Canadian Institutes of Health Research put out a request for proposals for research training programs, and the Institute of Infection and Immunity identified hepatitis $\mathrm{C}$ as a special area of interest, it became a 'natural' that we should collaborate across Canada to establish a training program in this area.

\section{WHEN REJECTION IS A GOOD THING}

Whereas a daily dose of rejection is not good for anyone, constructive criticism every now and again can be very helpful. Thus, it was that our second application for a research training program in hepatitis $\mathrm{C}$ was much more successful than the first. Our rejection slip included many good ideas such as covering the social and cultural aspects of infection with hepatitis C. This program now has 19 mentors and is truly multidisciplinary, covering nursing, sociology, behavioural medicine, aboriginal health, statistics and health economics in addition to the standard virology, immunology, vaccinology, infectious diseases and even hepatology.

\section{THE PHARMACEUTICAL INDUSTRY}

Good drugs will only get to market if a good clinical trial is conducted. Although in the past many industries have designed their own studies, they are increasingly involving the participating physicians. Although, for financial reasons, there is a distinct disadvantage for industry to work with universities, I believe the access to experienced academicians and lecturers is all to their advantage.

\section{INSTITUTION/UNIVERSITY}

Although it is unlikely that a Nobel Prize will ever be awarded to a clinical trialist, there are many reasons why the university is in favour of clinical trials. They provide a very fertile training ground for research for their students as well as promoting productive collaborations. Although many scientists are only interested in studying science for science's sake, there are others whose work needs to be translated to the clinical situation. These collaborations are sometimes the most exciting and the most rewarding.

\section{RESEARCH TRAINING EXPERIENCE}

From the point of view of the mentor, the studies with students, residents and fellows are often the most rewarding part of an academics career. Perhaps not always in the short term, but definitely in the long term! Although extremely timeconsuming, it is exhilarating to watch a trainee mature and then to start to teach the mentor. The trials and tribulations of trial conduct, analysis and publication are repeated by all generations; it never gets easier but it is always fun. Only rarely is a major discovery made, but it is important the students realize how their particular research study becomes a part of a much bigger picture. I liken research and the putting together of the data to an oil painting: the picture is never apparent in the beginning; it needs constant attention and realignment and examination both from a distance and close up; and only after a lot of work will it be complete. 
The most exciting part of clinical research or, for that matter, any research is the collaborations that are developed, both from within and outside one's department. These collaborations often lead to great friendships because they are based on a common desire and trust.

\section{CLINICAL TRIALS IN CHRONIC LIVER DISEASE - WHAT DO THEY ACHIEVE FOR THE PUBLIC?}

The past 30 years have seen a dramatic improvement in the management of liver disease. Probably most important is the development of the hepatitis $B$ vaccine, which can prevent the hepatitis B carrier state and its complications, most importantly hepatocellular carcinoma. At the other end of the spectrum, has been the development of liver transplantation for end stage liver disease. In between, prevention of disease and transplant are all the new therapies which have been particularly prolific in the area of chronic viral hepatitis. Now with the advent of nucleoside analogues, liver failure can be reversed, fatal flare ups of disease with intermittent chemotherapy can be prevented and safe liver transplantation can be performed in hepatitis $\mathrm{B}$ carriers. Cure for hepatitis $\mathrm{C}$ has become a very real possibility; up to $50 \%$ of those completing interferon and ribavirin therapy are cured. In those with progressive disease, liver cancer can be significantly reduced, if not entirely prevented. Doubtless in the next decade or so, most individuals with hepatitis $\mathrm{C}$ will be cured.

For other liver diseases, the picture is not so rosy. Although UDCA does delay progression of $\mathrm{PBC}$, we have yet to find a cure. To date, we still do not know how best to treat patients with PSC, although the other autoimmune liver disease, namely autoimmune hepatitis, can for the most part be well controlled although rarely cured. As we move into the 21 st century, nonalcoholic steatohepatitis becomes an ever increasing issue, both as a single entity and superimposed on other liver disease. The era of molecular genetics enables us to screen effectively for many liver diseases, and doubtless will facilitate specific diagnosis in the future. The future is at least as exciting as the past and I for one hope to be able to continue to participate in clinical research. 


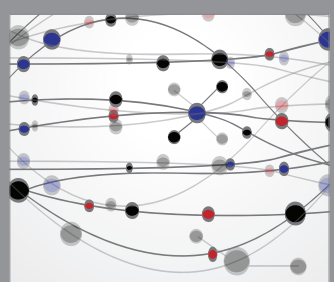

The Scientific World Journal
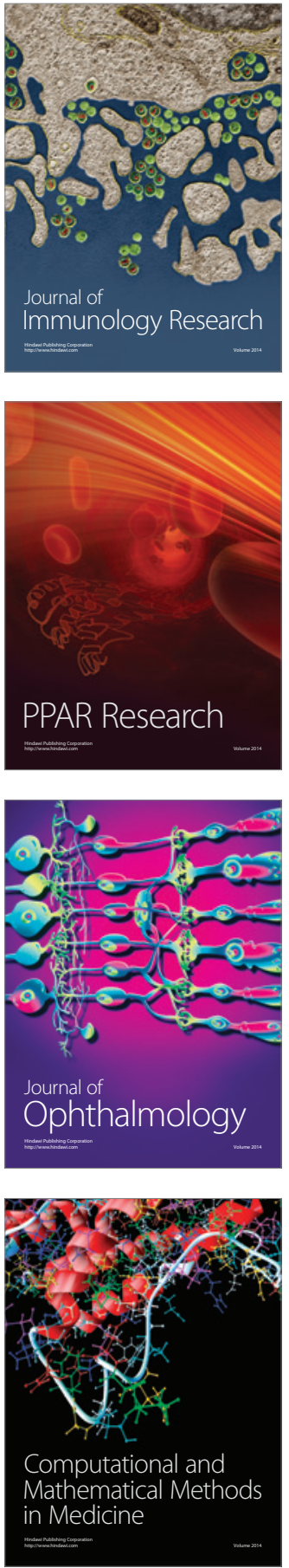

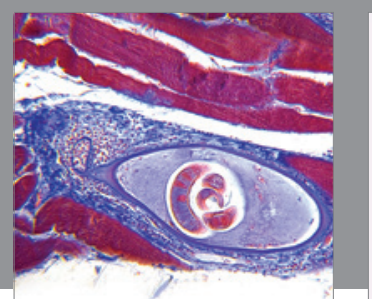

Gastroenterology Research and Practice

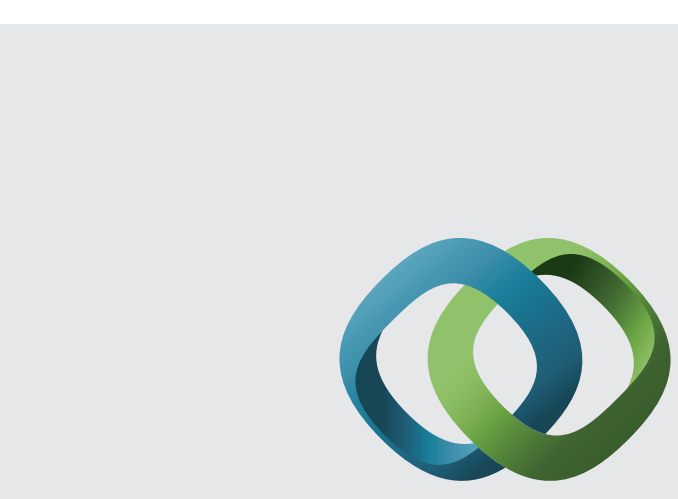

\section{Hindawi}

Submit your manuscripts at

http://www.hindawi.com
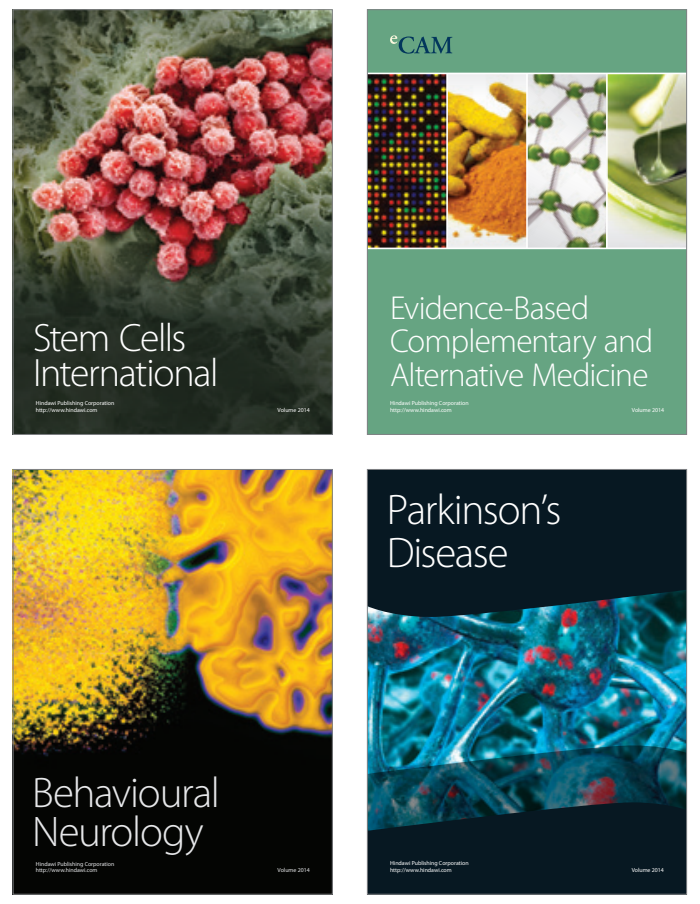
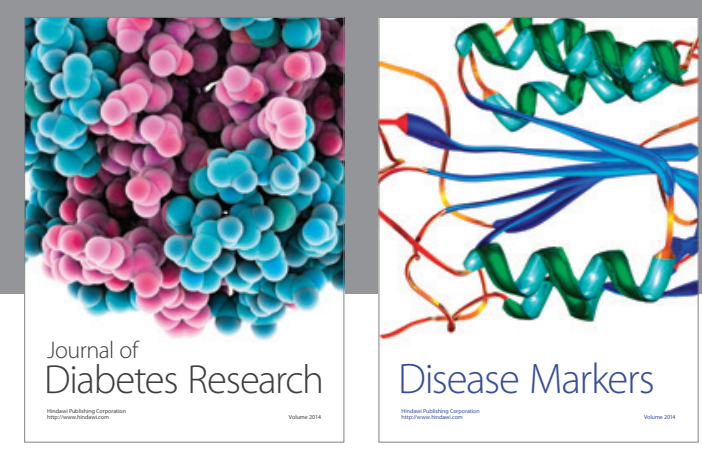

Disease Markers
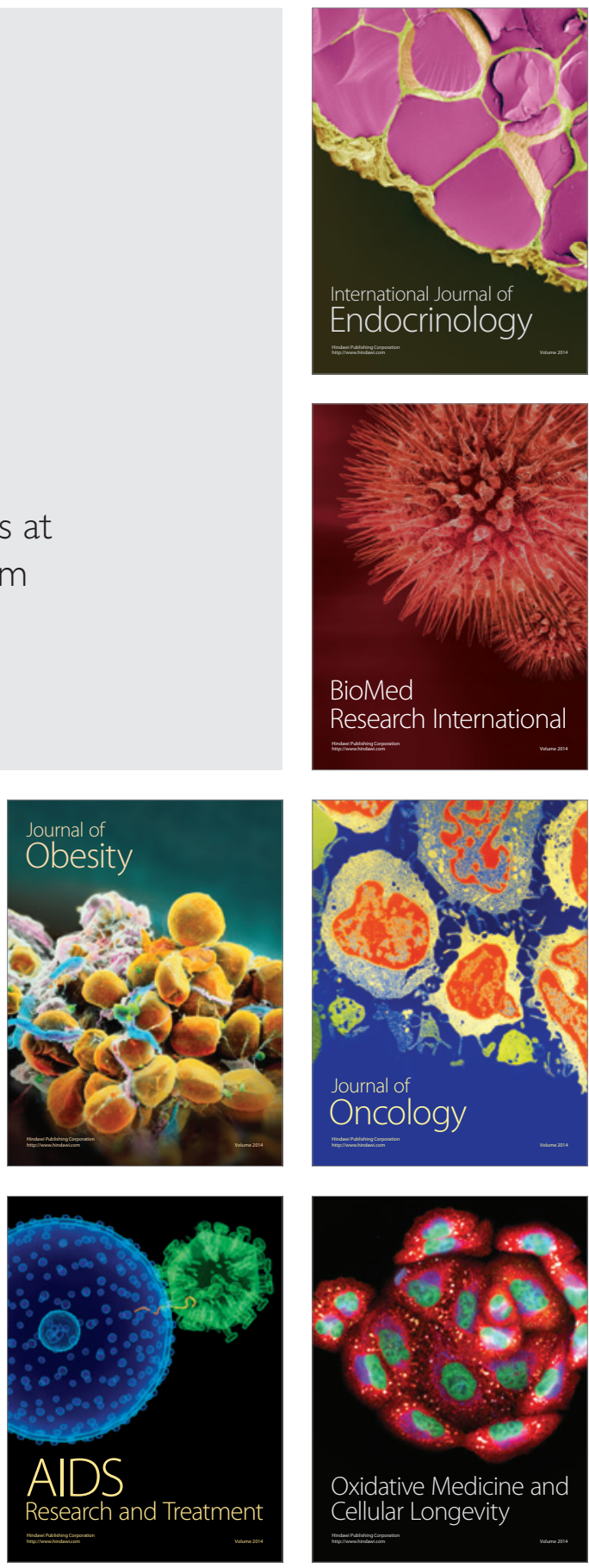